\title{
Die Verhältnismäßigkeit des Gewaltdarstellungsverbots
}

Über die Strafvorschrift des $\$ 131$ StGB wird seit jeher lebhaft diskutiert. Zumeist wird dabei die Bestimmtheit der Norm erörtert. Im folgenden Beitrag soll dagegen ihre Verhältnismäßigkeit behandelt werden, die bislang weniger im Mittelpunkt der Diskussion stand.

\section{Einleitung}

Das Gewaltdarstellungsverbot des $§ 131$ StGB, das bei seiner Einführung international und historisch ohne Vorbild war, ${ }^{1}$ ist auch heute noch sehr umstritten. ${ }^{2}$ Mehrfach ist der Vorschrift mangelnde Effizienz bescheinigt worden. ${ }^{3}$ Es wird beklagt, dass sich dies auch nach ihrer Erweiterung 1985 nicht wesentlich verändert hat. ${ }^{4}$ Verfassungsrechtliche Bedenken wurden vor allem unter dem Gesichtspunkt der Bestimmtheit (Art.103 Abs.2 GG) gegen die hohe Normativität der Tatbestandsumschreibung bzw. einzelner Tatbestandsmerkmale erhoben. ${ }^{5}$ Auch nach der »Tanz der Teufel «-Entscheidung des BVerfG, ${ }^{6}$ die $\$ 131$ StGB bei verfassungskonformer Auslegung als »noch hinreichend bestimmt « bezeichnet, ${ }^{7}$ wird der Tatbestand von der Rspr. teilweise sehr extensiv ausgelegt. ${ }^{8}$ Im folgenden sollen die Geeignetheit, die Erforderlichkeit und die Verhältnismäßigkeit im engeren Sinne des $\$ 131$ StGB geprüft werden, die bisher nicht ausreichend Beachtung gefunden haben.

1 Vgl. von Hartlieb, UFITA Bd. 86 (1980), 101; Greger, NStZ 1986, 8, 9; Meirowitz, Gewaltdarstellungen auf Videokassetten, 1993, S. 319; Ostendorf, in AK-StGB Bd. 3, 1986, §131 Rdnr. 2; Gehrhardt, NJW 1975, 375.

2 Vgl. Kühl, in Lackner/Kühl, StGB, 25. Aufl. 2004, §131 Rdnr. 1; von Bubnoff, in LKStGB, 11. Aufl. 1996, §131 Rdnr. 11; Ostendorf, (Fn. 1), §131 Rdnr. 5.

3 Vgl. etwa BT-Drs. 10/2546, S. 21; von Bubnoff, (Fn. 2), §131 Rdnr.1; Greger, (Fn. 1); Meirowitz, (Fn. 1), S. 320.

4 Vgl. etwa Kühl, (Fn. 2); von Bubnoff, (Fn. 2), §131 Rdnr.2; Joerden, ZRP 1995, 325; gegen die Annahme einer geringen Reichweite des §131 StGB Naujoks, ZRP 1999, 74; dagegen bezeichnet Meirowitz, (Fn. 1), S. 323 die Neufassung als schlagkräftiger.

5 Vgl. Kühl, (Fn. 2); von Bubnoff, (Fn. 2); Lenckner, in Schönke/Schröder, StGB, 26. Aufl. 2001, §131 Rdnr.2; Rudolphi, in SK-StGB, §131 Rdnr.2; Meirowitz, (Fn. 1), S. 345 ff; Ostendorf, (Fn. 1), §131 Rdnr. 5.

6 BVerfGE 87, 209 ff. = NJW 1993, $1457 \mathrm{ff}$.

7 Rudolphi, (Fn. 5) hält nur eine »sehr enge Interpretation « für möglich.

8 Kritisch dazu Beisel, AfP 1997, 514 f.; zu einer restriktiven Auslegung Köhne, GA 2004, $180 \mathrm{ff}$. 


\section{Die Verhältnismäßigkeit des §131 StGB}

Der verfassungsrechtliche Grundsatz der Verhältnismäßigkeit umfasst die Eignung und die Erforderlichkeit des vom Gesetzgeber gewählten Mittels. Zudem ist die Verhältnismäßigkeit im engeren Sinne zu prüfen.

\section{Geeignetheit}

Das vom Gesetzgeber eingesetzte Mittel ist geeignet, wenn mit seiner Hilfe der gewünschte Erfolg gefördert werden kann. ${ }^{9}$ Dabei soll nicht entscheidend sein, ob der erstrebte Erfolg tatsächlich eintritt; die bloße Möglichkeit der Zweckerreichung soll für die Eignung des Mittels ausreichen. ${ }^{10}$ Eine Ungeeignetheit ist danach nur anzunehmen, wenn das eingesetzte Mittel »objektiv« oder »schlechthin « ungeeignet ist. ${ }^{11} \mathrm{Ge}$ schütztes Rechtsgut des $§ 131$ StGB ist der »öffentliche Friede «. ${ }^{12}$ Dabei wird angenommen, dass Gewaltdarstellungen in Medien zu realen Gewalttätigkeiten durch die Rezipienten führen könnten. ${ }^{13}$ Diese Annahmen sind bis heute nicht eindeutig wissenschaftlich belegt; die Frage der Wirkungen medialer Gewalt ist weiter umstritten. ${ }^{14}$ Zumindest kurzfristige Wirkungen von Gewaltdarstellungen auf den Betrachter sind nicht sicher auszuschließen. ${ }^{15}$ Bei dem dargestellten engen Prüfungsmaßstab liegt es somit nahe, das Gewaltdarstellungsverbot trotz aller Uneinigkeit der Medienwirkungsforschung als jedenfalls nicht schlechthin ungeeignet zu bezeichnen. ${ }^{16}$ Dem Verhältnismäßigkeitsgrundsatz kommt aber gesteigerte Bedeutung für die Prüfung einer Strafvorschrift zu, die als schärfste dem Staat zur Verfügung stehende Sanktion ein sozial-ethisches Unwerturteil über ein bestimmtes Handeln des Bürgers ausspricht. ${ }^{17}$ Das strafbewehrte Verbot abstrakt gefährlichen Handelns ist zum Schutz des Rechtsguts nur geeignet, wenn die verbotenen Handlungsformen für das geschützte Rechtsgut typischerweise die naheliegende Möglichkeit einer Gefahr begründen; das gilt nicht für Handlungen, die etwa nur in Ausnahmesituationen dem Rechtsgut gefährlich sein können und nur eine vage, eher vom Zufall abhängige Möglichkeit einer Rechtsgütergefährdung begründen.$^{18}$ Danach ist es für strafbewehrte Verbote von Medien nicht ausreichend, dass in extremen Einzelfällen Gewaltdarstellungen als möglicherweise für reale Gewalttaten (mit-)ursächlich angesehen wurden: ${ }^{19}$ wenn das selbe Medium von

9 Vgl. BVerfGE 30, 292, 316; BVerfGE 33, 171, 187; BVerfGE 67, 157, 173.

10 Vgl. BVerfGE 67, 157, 175; BVerfGE 96, 10, 23; BVerfGE 103, 293, 307.

11 Vgl. BVerfGE 47, 109, 117; BVerfGE 81, 156, 192; BVerfGE 99, 341, 353.

12 Vgl. Lenckner, (Fn. 5), §131 Rdnr.1; Tröndle/Fischer, StGB, 52. Aufl. 2004, §131 Rdnr. 2; von Bubnoff, (Fn. 2), §131 Rdnr.9; Kühl, (Fn. 2).

13 Näher dazu SondA BT-Drs. 6/3521, S. 4 ff.

14 Näher zu den Theorien etwa Lober, CR 2002, 397 f.; auch unten 3.

15 So schon SondA BT-Drs. 6/3521, S. 5.

16 Vgl. Meirowitz, (Fn. 1), S. 360 und Jura 1993, 152, 154.

17 Vgl. BVerfGE 90, 145, 172.

18 Vgl. Graßhof, abweichende Meinung in BVerfGE 90, 199, 205.

19 So etwa im so genannten »Fall Jason« LG Passau NJW 1997, 1165 f.; dazu Eisenberg, NJW 1997, 1136 ff. 
einer Vielzahl von Personen völlig problemlos betrachtet wurde,${ }^{20}$ ist die Gewalthandlung im Einzelfall nicht als »typische« Gefahr für ein Rechtsgut anzusehen. Es ist als rechtlich zumindest sehr fragwürdig zu bezeichnen, die eindeutig überwiegende Mehrheit von problemlosen Fällen wegen ausgesprochen selten vorkommenden Problemfällen mit zu bestrafen. Vor der Schaffung einer Strafnorm ist es deshalb erforderlich, die Wahrscheinlichkeit einer Gefährdung des Rechtsguts realistisch einzuschätzen. Die Geeignetheit von Maßnahmen gegen eine Ursache, die für ein bestimmtes Problem in Betracht kommt, hängt zudem auch wesentlich davon ab, ob und inwieweit andere denkbar mögliche (Mit-)Ursachen behandelt werden. Je weniger bei einem multikausalen Phänomen gegen andere mögliche Ursachen getan wird desto mehr ist die Bekämpfung einer einzigen Ursache für den erstrebten Erfolg ungeeignet. Die neuere Medienwirkungsforschung lehnt ganz überwiegend die früheren monokausalen Erklärungsbemühungen ab. Es gibt keine direkten Wirkungen von (auch gewalthaltigen) Medieninhalten auf den Rezipienten. Mediale Gewaltdarstellungen stellen vielmehr einen Faktor neben vielen anderen, zum Teil wesentlich wichtigeren Faktoren dar. ${ }^{21}$ Die Eignung des $§ 131$ StGB zur Verhinderung von Gewalttätigkeiten muss somit im Zusammenhang mit Maßnahmen zur Bekämpfung anderer Ursachen von Gewaltstraftaten beurteilt werden. Eine isolierte Betrachtung ist nicht sinnvoll. Von besonderem Interesse ist insoweit der kriminologisch erheblich bedeutsame Faktor Alkohol. Es gehört seit langem zum kriminologischen Erfahrungswissen, dass Alkohol bei der Begehung vieler Straftaten zumindest »mit im Spiel « ist. ${ }^{22}$ Bezüglich regelmäßig wiederkehrender psychischer Faktoren der Tatsituation steht der Alkoholeinfluss deutlich an der Spitze, ${ }^{23}$ Alkohol ist als mitgestaltender Faktor der tatauslösenden Situation zu betrachten. ${ }^{24}$ Ein hoher Anteil der so genannten »Aggressionsdelikte « geht auf das Konto von alkoholisierten Tätern. ${ }^{25}$ Alkoholkonsum ist somit vielfach erheblich deutlicher und weniger umstritten als eine Ursache von (Gewalt-)Straftaten anzusehen als mediale Gewaltdarstellungen. In der Bundesrepublik Deutschland ist die Herstellung von alkoholischen Getränken nicht verboten. Die Abgabe ist nur gemäß §9 JuSchG an Kinder und Jugendliche beschränkt. ${ }^{26}$ Warnhinweise auf Flaschen mit alkoholischen Getränken werden als nicht erforderlich angesehen, da die Wirkungen des Alkohols im Kern zum allgemeinen Grundwissen gehören sollen. ${ }^{27}$ Ein Werbeverbot für Alkohol gibt es bislang nicht: ${ }^{28}$ im Fernsehen sind z.B. überaus häufig - insbesondere auch vor Sportveranstal-

20 Wie es wohl tatsächlich immer der Fall sein dürfte.

21 Näher Enquete-Kommission Zukunft der Medien BT-Drs. 13/11001, S. 15.

22 Vgl. Göppinger, Kriminologie, 5. Aufl. 1997, S. 594 f.

23 Vgl. Eisenberg, Kriminologie, 5. Aufl. 2000, §54 Rdnr. 8.

$24 \mathrm{Vgl}$ Kerner, in Kleines Kriminologisches Wörterbuch, 3.Aufl.1993, S. 8.

25 Vgl. von Hippel, ZRP 1999, 132, 133.

26 Diese bußgeldbewehrte ( $\$ 28$ Abs. 1 Nr. 10 und 11 JuSchG) Vorschrift ist aber wenig praxistauglich, da es faktisch »kinderleicht« ist, sie zu umgehen und als Minderjähriger an alkoholische Getränke zu gelangen.

27 So OLG Hamm NJW 2001, 1654, 1655.

28 Ob sich Pläne der zeitlichen Beschränkung von Werbung im Rundfunk tatsächlich durchsetzen lassen werden, ist angesichts zu erwartender massiver Kritik eher fraglich, vgl. dazu schon AfP 2002, $408 \mathrm{f}$. 
tungen - Werbe- bzw. Sponsoringspots von Herstellern alkoholischer Getränke zu sehen. Gegen den Konsum von Alkohol und damit die Gefahr seines Missbrauchs sowie die daraus resultierenden schweren Folgen wird somit derzeit praktisch nichts unternommen, obwohl es durchaus praktizierbare Maßnahmen gäbe. ${ }^{29}$ Dieses Nichteinschreiten gegen den nicht selten Kriminalität fördernden Alkoholkonsum muss bei der Beurteilung der Bekämpfung des (denkbaren) Nebenfaktors Gewaltdarstellungen entscheidend berücksichtigt werden. Gegen ein multikausales Problem wie Gewalttätigkeiten kann nur mittels einer alle möglichen Ursachen betreffenden Strategie effektiv vorgegangen werden. Maßnahmen gegen einen vergleichsweise wenig bedeutsamen Faktor können nichts zur Lösung beitragen, wenn zugleich deutlich wichtigere Faktoren nicht bekämpft werden. Ein einseitiger Lösungsversuch wird dem Problem der Gewalttätigkeiten und allgemein der Komplexität menschlicher Handlungsmotivationen nicht gerecht. Danach kann mit der Bekämpfung des relativ unwichtigen Faktors der medialen Gewaltdarstellungen - abgesehen von der berechtigten grundsätzlichen Skepsis gegenüber den Basisannahmen ${ }^{30}$ - das Ziel der Wahrung des öffentlichen Friedens nicht gefördert werden, solange wesentliche (Haupt-)Faktoren (wie z.B. der Alkoholmissbrauch) praktisch ungehindert fortwirken können. - Nach alledem ist $\$ 131 \mathrm{StGB}$ als nicht geeignetes Mittel zum Schutz des öffentlichen Friedens anzusehen. Insbesondere angesichts der fehlenden Maßnahmen gegenüber anderen Ursachen ${ }^{31}$ ist eine Zweckerreichung durch ihn nicht möglich. Das Gewaltdarstellungsverbot ist somit objektiv schlechthin ungeeignet. Die Erweiterung des Tatbestands auf »menschenähnliche Wesen« (BGB1. I 2003, 3007) ändert hieran nichts.

\section{Erforderlichkeit}

Das eingesetzte Mittel ist erforderlich, wenn der Gesetzgeber nicht ein anderes, gleich wirksames aber das Grundrecht nicht oder doch weniger fühlbar einschränkendes Mittel hätte wählen können. ${ }^{32}$ Der Gesetzgeber ist nur dort zur Wahl eines milderen Mittels verpflichtet, wo auch der weiter gehende Eingriff keinen besseren Erfolg verspricht. ${ }^{33}$ Verlangt wird eine eindeutig gleichwertige Alternative. ${ }^{34}$ Dabei ist es zunächst sehr problematisch, ob der $§ 131$ StGB überhaupt als effektives Mittel zur Bekämpfung realer Gewalt anzusehen ist. Ohne eine realistische Einschätzung der Effektivität des eingesetzten Mittels kann keine Gleichwertigkeit alternativer Lösungen geprüft werden. Bislang konnte in keinem Fall nachgewiesen werden, dass durch $\S 131$ StGB eine Gewaltstraftat verhindert wurde. Auch lässt sich nicht belegen, dass es ohne ihn zu (erheblich) mehr Gewaltstraftaten gekommen wäre. Offenbar steigt und sinkt die Gewaltkriminalität völlig unabhängig und unbeeinflusst von den beständig gesteigerten Beschränkungen des Medienkonsums Jugendlicher aber auch Erwachse-

29 Vgl. etwa von Hippel, (Fn. 25), 133 ff.

30 Näher dazu Köhne, NJW 50/2002, XVI.

31 Eine isolierte Bewertung der Geeignetheit des §131 StGB ist nicht sachgerecht.

32 Vgl. BVerfGE 30, 292, 316; BVerfGE 90, 145, 172; BVerfGE 92, 262, 273.

33 Vgl. BVerfGE 57, 250, 270.

34 Vgl. BVerfGE 30, 292, 319; BVerfGE 81, 70, 91. 
ner. Die Effektivität des $§ 131$ StGB ist daher sehr zweifelhaft. §131 StGB wird als nicht erforderlich angesehen, da der Jugendschutz im Bereich der Gewaltdarstellungen (heute durch das JuSchG) ausreichen müsse. ${ }^{35}$ In der Tat schränken die Jugendmedienschutzvorschriften die Verbreitung von Schriften auch an Erwachsene erheblich ein. ${ }^{36}$ Indizierte Bücher verschwinden vom Markt und sind auch für Erwachsene praktisch nicht vorhanden, da für sie nicht geworben werden darf. ${ }^{37}$ Der Jugendmedienschutz nimmt gewaltdarstellende Medien allerdings nicht völlig vom Markt und bewirkt keinen so wirksamen Erwachsenenschutz wie $\$ 131$ StGB ${ }^{38}$ Es ist aber höchst fraglich, ob ein »Erwachsenenschutz« überhaupt legitim ist. Dem Jugendschutz wird Verfassungsrang eingeräumt. ${ }^{39}$ Die Herleitung zum einen aus Art.5 Abs.2 GG zum anderen aus Art.6 Abs.2 GG lässt sich aber nicht auf Erwachsene übertragen. Auch die weniger überzeugende - Herleitung des Verfassungsrangs des Jugendschutzes aus Art.1 Abs.1 i.V. mit Art. 2 Abs. $1 \mathrm{GG}^{40}$ kann nicht zur Begründung eines »Erwachsenenschutzes « herangezogen werden, da sie auf die Schutz- und Hilfebedürftigkeit des Minderjährigen zur Entwicklung zu einer eigenverantwortlichen Persönlichkeit innerhalb der sozialen Gemeinschaft abstellt. ${ }^{41}$ Der »Erwachsenenmedienschutz « genießt daher keinen Verfassungsrang. Seine Legitimität hängt deshalb wesentlich von der Wirkung medialer Gewaltdarstellungen auf Erwachsene ab: nur wenn (zumindest) in den meisten Fällen von einer gewaltfördernden Wirkung auszugehen wäre, bedürfte es eines Erwachsenenschutzes und berechtigte dieser zur allgemeinen Beschränkung der Grundrechte aus Art.5 Abs.1 GG. Hier ist zunächst zu beachten, dass sich Wirkungsstudien meist auf Kinder und Jugendliche beziehen. ${ }^{42}$ Eine Übertragbarkeit der Ergebnisse auf Erwachsene ist wegen der regelmäßig stärkeren Beeinflussbarkeit sich in der Entwicklung befindlicher Minderjähriger nicht ohne weiteres möglich. Zudem liegen keine eindeutigen Resultate vor: die Ergebnisse der Wirkungsforschung sind - auch bei Kindern und Jugendlichen - weiterhin umstritten. ${ }^{43}$ Es genügt insoweit nicht, dass zumindest kurzfristige Wirkungen auf die Rezipienten nicht sicher auszuschließen sind und Gewaltdarstellungen aggressionsfördernd wirken können, wenn sie beim Betrachter auf bestimmte Prädispositionen treffen. ${ }^{44}$ Eine nicht sicher auszuschließende Wirkung bedeutet bei weitem keine positive Bestätigung der Annahme und reicht angesichts der absolut herausragenden Bedeutung der Grundrechte aus Art.5 Abs.1 GG ${ }^{45}$

35 Vgl. Ostendorf, (Fn. 1), §131 Rdnr. 6.

36 So zu »schwer« jugendgefährdenden Werken BVerfGE 83, 130, 144; auch BVerfGE 90, 1, 16.

37 Vgl. Naumann, in Dankert/Zechlin, Literatur vor dem Richter, 1988, 195, $198 \mathrm{f}$.

38 Vgl. Meirowitz, (Fn. 1), S. 361 und Jura 1993, 152, 154.

39 Vgl. etwa BVerfGE 30, 336, 347 f.; BVerfGE 77, 346, 356; BVerfGE 83, 130, 139.

40 Vgl. BVerfGE 83, 130, 140.

41 Dabei ist schon stark zu bezweifeln, ob die generelle massive Beschränkung des Medienkonsums von Kindern und Jugendlichen durch staatliche Maßnahmen die Herausbildung eines selbstbewussten, eigenbestimmten Individuums wirklich fördern kann.

42 Worauf auch Meirowitz, (Fn. 1), S. 359 und Jura 1993, 152, 154 hinweist.

43 Vgl. Lober, (Fn. 14).

44 So SondA BT-Drs. 6/3521, S. 5.

45 Hierzu näher BVerfGE 5, 85, 205; BVerfGE 7, 198, 208; BVerfGE 20, $56,97$. 
nicht zu deren Beschränkung durch eine Strafvorschrift aus. Dass Gewaltdarstellungen auf bestimmte Prädispositionen beim Rezipienten treffen müssen, um eine aggressionsfördernde Wirkung haben zu können, verdeutlicht, dass eine Wirkung in der ganz überwiegenden Zahl bei Erwachsenen (eben alle ohne entsprechende ungünstige Veranlagungen) nicht eintreten wird und deshalb keine regelmäßige gewaltfördernde Wirkung auf Erwachsene anzunehmen ist. Neuere Untersuchungen zur Medienwirkungsforschung ${ }^{46}$ haben ergeben, dass es keine direkten Wirkungen von medialen (Gewalt-)Darstellungen auf den Betrachter gibt und Gewaltdarstellungen nur einen Faktor neben vielen anderen, zum Teil wesentlich wichtigeren Faktoren darstellen. ${ }^{47}$ Auch dies zeigt, dass i.d.R. keine Gewaltförderung durch Gewaltdarstellungen stattfindet. Bestätigt wird dies dadurch, dass Erwachsene im Ausland ohne eine $\$ 131$ StGB vergleichbare Vorschrift (etwa Frankreich oder die Niederlande) nicht deswegen, d.h. weil sie Gewaltdarstellungen, die in Deutschland verboten sind, betrachtet haben, erheblich häufiger (Gewalt-)Straftaten begehen. ${ }^{48}$ Gegen die Annahme einer regelmäßigen Gewaltförderung bei Erwachsenen spricht schließlich, dass ansonsten sämtliche Mitglieder von Medienkontrollgremien (etwa der Bundesprüfstelle für jugendgefährdende Medien oder der FSK) extrem gefährdet wären, durch ihre Tätigkeit zu aggressiven (Straf-)Taten animiert zu werden. Es ist auch nicht nachvollziehbar, Erwachsene einerseits durch Wahlen die Regierung des Staates (mit-)bestimmen zu lassen, sie aber andererseits für durch die Medien derart leicht beeinflussbar zu halten, dass sie mittels einer Strafvorschrift vor dem Konsum gewisser Inhalte »geschützt« werden müssten. Da nach alledem statt von einer regelmäßigen gewaltfördernden Wirkung medialer Darstellungen auf Erwachsene eher davon auszugehen ist, dass eine solche nur in extrem seltenen Ausnahmefällen beim Zusammentreffen mit ungünstigen Vorbedingungen denkbar möglich ist, ist ein »Erwachsenenmedienschutz« nicht legitim. Da ein Erwachsenenschutz somit nicht zu rechtfertigen ist, muss der Gesetzgeber sich auf den Jugendmedienschutz beschränken. Dieser ist (auch) hinsichtlich Gewaltdarstellungen durch das JuSchG völlig hinreichend geregelt. ${ }^{49}$ Die Effektivität des $§ 131$ StGB ist danach höchst fraglich; der einzige begründbare Zweck ${ }^{50}$ - der Jugendschutz - wird durch das JuSchG so weit als möglich erreicht. Der weiter gehende Eingriff durch $\$ 131$ StGB ist daher (insbesondere bei Einbeziehung auch »menschenähnlicher Wesen«) nicht erforderlich.

46 Die wiederum zumeist auf Minderjährige bezogen sind.

47 Vgl. Enquete-Kommission Zukunft der Medien BT-Drs. 13/11001, S. 15.

$48 \mathrm{Zu}$ Jugendlichen und der Notwendigkeit eines gemeinsamen europäischen Jugendmedienschutzes vgl. Köhne, MMR 9/2002, XXI ff.

49 Ansonsten würde sich zwangsläufig die - hier nicht näher zu behandelnde - Frage nach der Geeignetheit und Erforderlichkeit des JuSchG stellen.

50 Wobei auch hier Zweifel an der Wirksamkeit strenger Vorschriften angebracht sind. 


\section{Verhältnismäßigkeit im engeren Sinne}

Die dritte Stufe des Verhältnismäßigkeitsgrundsatzes, die Verhältnismäßigkeit im engeren Sinne, die auch als »Angemessenheit $~_{51}$ oder als $\gg$ Zumutbarkeit ${ }^{52}$ bezeichnet wird, setzt gegenüber den ersten beiden Stufen weniger auf rationale Maßstäbe, wenn sie nach der Zumutbarkeit und Angemessenheit fragt. Sie dient letztlich in erster Linie der Korrektur offensichtlich unhaltbarer Ergebnisse. ${ }^{53}$ Die mit der Regelung verfolgten Zwecke dürfen nicht außer Verhältnis zu der Belastung des Bürgers stehen. ${ }^{54}$ Der durch die Regelung bewirkte Eingriff in das Grundrecht des Bürgers muss in angemessenem Verhältnis zu dem mit ihr verfolgten Zweck stehen. ${ }^{55}$ Verlangt wird eine Abwägung zwischen Gemeinwohlbelangen, zu deren Wahrnehmung es erforderlich ist, in Grundrechte einzugreifen, und den Auswirkungen auf die Rechtsgüter der davon Betroffenen. ${ }^{56}$ Die Geeignetheit und Erforderlichkeit des $\$ 131$ StGB einmal angenommen, ${ }^{57}$ müsste der durch ihn bewirkte Eingriff in die Grundrechte aus Art.5 Abs.1 GG in einem angemessenen Verhältnis zur mit ihm beabsichtigten Bewahrung des öffentlichen Friedens ${ }^{58}$ stehen. Präzise ausgedrückt ist mit $\$ 131$ StGB die Bewahrung des öffentlichen Friedens durch die Verhinderung einer Förderung von Aggressionsbereitschaft zum Schutz der Allgemeinheit und jedes Einzelnen vor Gewalttaten bezweckt. ${ }^{59}$ Der Schutz des Einzelnen und der Allgemeinheit ist ein Gut von essentieller Bedeutung. ${ }^{60}$ Auch die betroffenen Grundrechte aus Art.5 GG sind von hohem Rang für ein staatliches Gemeinwesen. ${ }^{61}$ Gefordert wird eine Abwägung der widerstreitenden Belange; es ist dabei nicht möglich, einem der Belange grundsätzlichen Vorrang einzuräumen. ${ }^{62}$ Es liegt aber nahe, den Schutz vor Gewalttaten regelmäßig der Freiheit Erwachsener, die sie interessierenden Medieninhalte auszuwählen, oder der Film- oder Kunstfreiheit vorzuziehen. Hierfür spräche schon die Einschränkbarkeit der Grundrechte aus Art.5 Abs.1 GG durch die Schranken des Art.5 Abs.2 GG. Auch lässt sich vertreten, dass $§ 131$ StGB die Grundrechte aus Art.5 GG nur in einem kleinen Bereich (massive Gewaltdarstellungen) einschränkt, der ohnehin wenig schützenswert erscheint. ${ }^{63}$ Allerdings berührt dies eher die Frage, ob überhaupt ein zu rechtfertigender Eingriff in ein Grundrecht vorliegt. Entscheidend ist letztlich aber die Wahrscheinlichkeit der Verletzung der geschützten Belange. Die Verhältnismäßigkeit im engeren Sinne kann im Falle des Gewaltdarstellungsverbots nur dann bejaht wer-

51 Vgl. etwa BVerfGE 93, 213, 237 f.; BVerfGE 100, 313, 375 f.

52 Vgl. etwa BVerfGE 30, 292, 316; BVerfGE 102, 1, 20.

53 So Graßhof, (Fn. 18), 200.

54 Vgl. BVerfGE 50, 217, 227; BVerfGE 80, 103, 107.

55 Vgl. BVerfGE 99, 202, $212 \mathrm{f}$.

56 Vgl. BVerfGE 92, 277, 327.

57 Entgegen der oben vertreten Auffassung, vgl. oben 1. und 2.

58 Vgl. Nachweise Fn. 12.

59 Vgl. Maurach/Schroeder/Maiwald, Strafrecht BT Teilbd. 2, 8. Aufl. 1999, § 94 Rdnr. 2.

60 Vgl. Meirowitz, (Fn. 1), S. 361.

61 Vgl. Meirowitz, (Fn. 1), S. 362; näher dazu BVerfG (Fn. 45).

62 Vgl. BVerfGE 83, 130, 143.

63 In diesem Sinne Meirowitz, (Fn. 1), S. 363. 
den, wenn die Gefährdung von Leben oder Gesundheit aufgrund einer durch bestimmte Medieninhalte bewirkten Aggressionsförderung bei Erwachsenen (zumindest) annähernd so wahrscheinlich erscheint wie der Eingriff in Grundrechte aus Art.5 GG durch §131 StGB. Ansonsten wäre es denkbar, dass das durch Grundrechte geschützte Verhalten Erwachsener mittels einer Strafvorschrift wegen einer tatsächlich (sehr) fernliegenden Möglichkeit der Verletzung der Rechtsgüter anderer eingeschränkt würde. Durch $\S 131$ StGB wird in jedem Fall in Grundrechte aus Art.5 GG eingegriffen: zu denken ist hier insbesondere an die Filmfreiheit und die Kunstfreiheit, ${ }^{64}$ auch die Freiheit der Meinungsäußerung ist betroffen, weil nicht selten durch massive Gewaltdarstellungen in Filmen (auch) eine bestimmte Einsicht oder Auffassung vermittelt werden soll und weil es daneben in der Bundesrepublik Deutschland faktisch (nahezu) unmöglich ist, sich überhaupt eine Meinung über bestimmte Filme zu bilden, da diese nicht ungeschnitten erhältlich sind. ${ }^{65}$ Auch kann ein Verstoß gegen das Zensurverbot ${ }^{66}$ angenommen werden, da sich inländische Filmhersteller bzw. -vertreiber durch $§ 131$ StGB zu einer (Selbst-)Zensur gezwungen sehen. ${ }^{67}$ Diesen immer stattfindenden Eingriffen in Grundrechte durch \$131 StGB müsste eine empirisch nachweisbare (nahezu) ebenso hohe Wahrscheinlichkeit der Ausübung von aggressiven Handlungen aufgrund exzessiver medialer Gewaltdarstellungen gegenüberstehen. Das Betrachten von Gewaltdarstellungen müsste also praktisch fast immer zu realen Gewalttätigkeiten durch den Rezipienten führen. Bei aller Uneinigkeit der Medienwirkungsforschung hinsichtlich der Wirkungstheorien ${ }^{68}$ kann jedenfalls nicht behauptet werden, dass mediale Gewalt (fast) immer zu einer Aggressionssteigerung bei jedem Rezipienten führt. Diese These würde durch - insbesondere auch internationale - Erfahrungen entkräftet. Sie widerspräche zudem dem auch von Befürwortern des $§ 131$ StGB eingeräumten Umstand, dass es zur aggressionssteigernden Wirkung von Gewaltdarstellungen bestimmter Prädispositionen beim Betrachter oder anderer äußerer Bedingungen bedarf. ${ }^{69}$ Außerdem würde sie die Individualität des jeweiligen Betrachters, der abhängig von seiner individuellen Persönlichkeit und seinen eigenen Erfahrungen auf die Darstellungen völlig unterschiedlich reagieren kann, außer Acht lassen. Es gibt sicherlich reichlich Personen, bei denen i.S. der Katharsistheorie durch das Betrachten (oder gar Mitvollziehen) von dargestellten Gewaltakten die Bereitschaft zu eigenem aggressiven Handeln abnimmt. Auch werden viele Menschen i.S. der Inhibitionstheorie durch die Beobachtung von gewalttätigen Verhaltensweisen Angst vor Aggressionen empfinden und dadurch selbst weniger aggressiv agieren. Denkbar ist aber ebenfalls, dass bei manchen i.S. der Stimulationstheorie durch das Betrachten von Gewaltdarstellungen die Bereitschaft zu eigenen aggressiven Handlungen erhöht wird.

64 Hierzu näher Meirowitz, (Fn. 1), S. 350 ff., S. 372 ff. und Jura 1993, 152 ff.

$65 \mathrm{Zu}$ kritischen Beiträgen über indizierte Filme vgl. BGHSt 34, $218 \mathrm{ff}$.

66 Kein Grundrecht sondern nach h.M. eine Eingriffsschranke, vgl. BVerfGE 33, 52 Ls. 4b.

67 Bekannt als so genannte »Schere im Kopf«.

68 Kritisch zu den vom Gesetzgeber bei Einführung des $\$ 131$ StGB vorgezogenen und bis heute nicht relativierten Basisannahmen oben 1. und 2.

69 Vgl. SondA BT-Drs. 6/3521, S. 5; von Bubnoff, (Fn. 2), §131 Rdnr. 8; Glogauer, ZRP $1990,376,380$. 
Auch erscheint es möglich, dass bei einigen Personen i.S. der Habitualisierungstheorie durch dauerhafte Konfrontation mit Gewaltdarstellungen eine Gewöhnung an die fiktive Gewalt und dadurch eine gesteigerte Neigung zu eigener tatsächlicher Gewalt eintritt. Einigkeit besteht in der derzeitigen Medienwirkungsforschung lediglich darüber, dass es keine direkten Wirkungen von Medieninhalten auf den Zuschauer gibt. ${ }^{70}$ Letztlich werden wohl tatsächlich sämtliche Theorien als annehmbar weil bei verschiedenen Menschen zutreffend erscheinen. Eine sämtliche Rezipienten hinsichtlich ihrer Reaktionsweise auf (gewalthaltige) Medieninhalte erfassende Wirkungstheorie ist danach nicht realistisch. Auch dürfte es unmöglich sein, die Zugehörigkeit von Zuschauern zu den genannten Theorien zahlenmäßig zuverlässig zu erfassen (denkbar ist z.B., dass ein einzelner Mensch von der einen Gewaltdarstellung abgestoßen und dadurch von tatsächlicher Gewalt abgebracht wird, eine andere aber seine Bereitschaft dazu erhöht). Jedenfalls ist aber eine Aggressionsförderung durch Gewaltdarstellungen nicht einmal annähernd in sämtlichen Fällen des Betrachtens solcher Medieninhalte anzunehmen. Realistisch gesehen beschränken sich Fälle von Gewalthandlungen, in denen die vorherige Rezeption gewalthaltiger Medien möglicherweise als (mit-)ursächlich anzusehen ist, auf ausgesprochen wenige, von Presse und Fernsehen aber viel beachtete Einzelfälle, ${ }^{71}$ während die regelmäßige folgenlose Betrachtung durch die ganz überwiegende Zahl von Zuschauern nicht erwähnt wird, weil sie für die Medienberichterstattung nicht spektakulär genug ist. Danach ist die Gefahr der Verletzung von Leben, Leib oder Gesundheit anderer durch den Konsum bestimmter Medien deutlich weniger wahrscheinlich als die stets durch $\$ 131$ StGB stattfindenden Eingriffe in Grundrechte aus Art.5 GG. Somit stehen die mit $\$ 131$ StGB verfolgten Zwecke außer Verhältnis zu den durch ihn bewirkten Beeinträchtigungen von (Grund-)Rechten des Bürgers. Es ist nicht angemessen, wegen einer bloß theoretisch angenommenen und ohnehin allenfalls in Ausnahmefällen im Zusammenwirken mit weiteren notwendigen Faktoren denkbaren Möglichkeit der Aggressionsförderung durch Gewaltdarstellungen der ganz eindeutig überwiegenden Mehrheit folgenlos zuschauender Bürger den Umgang mit solchen Medieninhalten zu beschränken. Zu bedenken ist insoweit auch, dass die Verbote des $\$ 131$ StGB zu erheblichen Umsatzeinbußen bei Herstellern und Vertreibern der Medien sowie bei kleineren Firmen zur Vernichtung ihrer wirtschaftlichen Existenz führen können. Die allgemeine Beschränkung von Grundrechten aus Art.5 GG wegen der wissenschaftlich nicht sicher auszuschließenden Möglichkeit einer Förderung der Gewaltbereitschaft durch gewalthaltige Medien bei Einzelnen, die zudem eines Zusammentreffens mit anderen ungünstigen Umständen bedarf, ist nicht zumutbar. Dem nicht durch Medien zu realer Gewalttätigkeit anregbaren Zuschauer kann der Konsum von Gewaltdarstellungen nicht wegen einzelner Personen, die auf die dargestellte Gewalt atypisch reagieren, begrenzt oder gar untersagt werden. Es erscheint grundsätzlich nicht legitim, einer klaren Mehrheit von Personen Nachteile aufzuerlegen weil eine verhältnismäßig sehr kleine (Ziel-)Minderheit dadurch auch betroffen wäre. Dies lässt sich an einem einfachen Beispiel verdeutlichen: Die vereinzelt

70 Vgl. Enquete-Kommission Zukunft der Medien BT-Drs. 13/11001, S. 15.

71 Wie etwa den so genannten »Fall Jason«, vgl. Fn. 19. 
(nicht immer unbedingt ernsthaft) erhobene Forderung einer nächtlichen Ausgangssperre für sämtliche Männer zum Schutz von Frauen vor einer Vergewaltigung ist offenkundig ungerechtfertigt und überzogen weil die ganz überwiegende Zahl der von einer solchen Maßnahme Betroffenen ohnehin keine Sexualdelikte begeht. Die Belastung der eindeutig überwiegenden Zahl unauffällig bleibender, d.h. nicht aggressiv werdender Konsumenten gewalthaltiger Medien durch die erhebliche Erschwerung (bzw. oftmals gar Verhinderung) ihrer Erlangung kann deshalb diesen nicht zugemutet werden. -Danach ist die Verhältnismäßigkeit im engeren Sinne hinsichtlich des $\$ 131$ StGB zu verneinen. Insbesondere der Umstand, dass die Zahl der völlig ungerechtfertigt Belasteten gegenüber der Zahl der tatsächlich rechtmäßig (weil aufgrund Medienkonsums »gefährlicher«) Betroffener klar überwiegt, schließt die Verhältnismäßigkeit einer allgemeinen Strafdrohung aus.

\section{Ergebnis und Schluss}

Da nach der derzeitigen wissenschaftlichen Einschätzung die Beschäftigung mit gewaltdarstellenden Medien allenfalls als ein minimaler Faktor für reale Gewalttätigkeiten angesehen werden kann und andere vielfach wesentlich wichtigere Faktoren (etwa Alkohol) faktisch nicht behandelt werden, ist die Beschränkung des Medienkonsums durch eine Strafvorschrift ( $\$ 131$ StGB) nicht zur Wahrung des öffentlichen Friedens geeignet. ${ }^{72}$ Ein Erwachsenenschutz vor gewissen Medieninhalten lässt sich mangels hinreichend deutlicher und eindeutiger Erkenntnisse der Medienwirkungsforschung nicht rechtfertigen. Der Jugendmedienschutz wird durch das JuSchG auch bezüglich Gewaltdarstellungen bereits ausreichend und durchaus auch mit begrenzender Wirkung auf den Medienkonsum Erwachsener geregelt. Eine weiter gehende Erstreckung des Schutzes durch eine Vorschrift des allgemeinen Strafrechts ist deshalb nicht erforderlich. ${ }^{73}$ Bei der Prüfung der Verhältnismäßigkeit im engeren Sinne kommt es (zumindest bei Strafvorschriften) entscheidend darauf an, ob eine Verletzung des durch die Regelung zu schützenden Rechtsguts (wenigstens annähernd) so wahrscheinlich ist wie die Verletzung des andererseits betroffenen Rechtsguts. Ansonsten würden sich allgemeingültige Beschränkungen mit vereinzelten, atypischen Ausnahmefällen rechtfertigen lassen. Im Rahmen des $§ 131$ StGB ist es trotz vieler Untersuchungen nicht gelungen, hinreichend zu belegen, dass der Konsum bestimmter Medien (fast) immer zu realer Gewalt führt. Die Verletzung von Leben, Leib oder Gesundheit einer Person aufgrund der Rezeption eines gewalthaltigen Mediums ist danach bei weitem nicht so wahrscheinlich wie die durch $\$ 131$ StGB in jedem Fall stattfindende Verletzung von Grundrechten aus Art.5 GG. §131 StGB ist somit nicht verhältnismäßig im engeren Sinne. Die Beschränkungen durch das Gewaltdarstellungsverbot sind insbesondere angesichts der sehr stark zu bezweifelnden Wirksamkeit des $§ 131$ StGB zur Verhinderung von Gewalttätigkeiten nicht angemessen und nicht zumutbar. ${ }^{74}$ Damit ist $§ 131$

72 Vgl. oben II. 1.

73 Vgl. oben II. 2.

74 Vgl. oben II. 3. 
StGB auf allen drei Stufen als nicht verhältnismäßig anzusehen. Er ist daher verfassungswidrig und sollte deshalb ersatzlos gestrichen werden. Auch die Erweiterung des Tatbestands auf »menschenähnliche Wesen «, ${ }^{75}$ deren hinreichende Bestimmtheit i.S. des Art.103 Abs.2 GG höchst zweifelhaft erscheint, ${ }^{76}$ ändert an diesem Ergebnis nichts, sondern verdeutlicht die Ungeeignetheit eher noch: Die Medienwirkungsforschung belegt nämlich keineswegs, dass dargestellte Gewaltaktionen gegen fiktive nichtmenschliche Wesen $\mathrm{zu}$ einer Gewaltförderung führen, sondern legt vielmehr nahe, dass der Betrachter auf realitätsferne Darstellungen weniger sensibel und betroffen reagiert. ${ }^{77}$ Eine neuere stichprobenartige Befragung Jugendlicher kommt ${ }^{78} \mathrm{zu}$ dem Ergebnis, dass nicht jeder Rezipient gewalthaltiger Medien gewalttätig sein muss, aber dass wohl viel eher eine gewaltbereite Person derartige Medien gerne konsumiert. ${ }^{79}$ Beim gesamten Jugendmedienschutz und noch erheblich verstärkt bei dem auch Erwachsene betreffenden $\$ 131$ StGB wird daher Ursache und Wirkung verkehrt und zudem entgegen der ganz überwiegenden neueren Medienwirkungsforschung weiterhin (Mono-)Kausalität behauptet. Nach alledem ist deswegen der $\$ 131$ StGB über 30 Jahre nach seiner Einführung ${ }^{80}$ aufzuheben. Ohne hinreichend sichere Erkenntnisse über eine Gemeinschädlichkeit darf der Medienkonsum Erwachsener nicht beschränkt werden.

75 Vgl. hierzu BT-Drs. 15/1311, S. 21 f.

76 Hierzu näher Köhne, (Fn. 8), 182 f.

77 So zu Minderjährigen Enquete-Kommission Zukunft der Medien BT-Drs. 13/11001, S. 17 f.; für Erwachsene gilt dies noch mehr.

78 Unter deutlichem Hinweis darauf, dass aus der Feststellung empirisch beobachteter Zusammenhänge keine Kausalaussagen zu ziehen sind.

79 Vgl. Raithel, MSchrKrim 2003, 287, 296.

80 Vgl. BGBl. I 1973, 1725 f. 